\title{
Prevalencia de la osificación heterotópica tras artroplastia de disco cervical a los 5 años
}

\section{Prevalence of Heterotopic Ossification after Cervical Disk Arthroplasty at 5 Years}

\author{
Ivan A. Stepanov ${ }^{1}$ (ㄴ) Vladimir A. Beloborodov ${ }^{1}$ \\ ${ }^{1}$ Departamento de Cirugía General y Anestesiología, Irkutsk State \\ Medical University, Irkutsk, Russia \\ Address for correspondence Ivan A. Stepanov, MD, Krasnogo \\ Vosstaniya Str. 1, 664003, Irkutsk, Irkutskaya Oblast', Russia \\ (e-mail: stepanovivanneuro@gmail.com).
}

Rev Chil Ortop Traumatol 2021;62(3):e174-e179.

\section{Resumen \\ Palabras Clave \\ - discectomía y fusión cervical anterior \\ - artroplastia de disco cervical \\ - osificación heterotópica \\ - prevalencia \\ - prótesis Activ C \\ - prótesis M6-C}

Introducción En los últimos años, la artroplastia de disco cervical (ADC) se ha utilizado ampliamente en pacientes como alternativa a la disectomía y fusión cervical anterior (DFCA). Sin embargo, se han informado osificación heterotópica $(\mathrm{OH})$ y fusion espontánea después de $\mathrm{ADCs}$, y el desarrollo de $\mathrm{OH}$ puede dificultar el mantenimiento de movimiento después de la artroplastia.

Materiales y Métodos El procedimiento ADC con prótesis Activ Cy M6-C se realizó en 127 pacientes. El tiempo medio de seguimiento fue de 58.4 meses, con un rango de 51 a 66 meses.

Resultados Las osificaciones de grado 1 estuvieron presentes en 11 niveles (8,6\%). Un total de $45(35,4 \%)$ segmentos eran de $\mathrm{OH}$ de grado 2 . Las $\mathrm{OHs}$ que produjeron restricciones del rango de movimiento estuvieron presentes en $13(10,2 \%)$ casos. A los 5 años de seguimiento, sólo había $9(7,0 \%)$ pacientes con osificación de grado 4 en el grupo de prótesis de disco artificial M6-C. En el análisis de la supervivencia tras la ocurrencia de $\mathrm{OH}$, la mediana de los pacientes fue de 28,3 $\pm 5,6$ meses. El grupo de prótesis de disco artificial Activ $C$ tuvo una supervivencia estadísticamente más larga (49,5 $\pm 7,8$ meses) que el grupo de disco M6-C.

Conclusiónes En este estudio, el 61,4\% de los pacientes desarrollaron $\mathrm{OH}$ en un período de seguimiento medio de 58,4 meses. En el análisis de la supervivencia tras la ocurrencia de $\mathrm{OH}$, la mediana de los pacientes fue de $28.3 \pm 5.6$ meses. El grupo de prótesis de disco artificial Activ $C$ tuvo una supervivencia estadísticamente más larga $(49.5 \pm 7.8$ meses) que el grupo de disco M6-C. recibido

03 de junio de 2020

aceptado

06 de agosto de 2021
DOI https://doi.org/

10.1055/s-0041-1740171. ISSN $0716-4548$. (c) 2021. Sociedad Chilena de Ortopedia y Traumatologia. All rights reserved.

This is an open access article published by Thieme under the terms of the Creative Commons Attribution-NonDerivative-NonCommercial-License, permitting copying and reproduction so long as the original work is given appropriate credit. Contents may not be used for commercial purposes, or adapted, remixed, transformed or built upon. (https://creativecommons.org/ licenses/by-nc-nd/4.0/)

Thieme Revinter Publicações Ltda., Rua do Matoso 170, Rio de Janeiro, RJ, CEP 20270-135, Brazil 


\begin{abstract}
Keywords

- anterior cervical diskectomy and fusion

- cervical disk arthroplasty

- heterotopic ossification

- prevalence

- activ C prosthesis

- M6-C prosthesis

Introduction In recent years, cervical disk arthroplasty (CDA) has become widely used in patients as a substitute to anterior cervical diskectomy and fusion (ACDF). However, heterotopic ossification ( $\mathrm{HO}$ ) and spontaneous fusion after CDA have been reported, and maintenance of motion following arthroplasty can be hindered by the development of HO.

Materials and Methods The CDA procedure with Activ $C$ and M6-C prostheses was performed on 127 patients. The mean follow-up time was of 58.4 months, ranging from 51 to 66 months.

Results Grade- 1 ossifications were present in 11 (8.6\%) levels. A total of 45 (35.4\%) segments showed grade- $2 \mathrm{HO}$. Cases of $\mathrm{HO}$ that led to restrictions in the range of motion were present in $13(10.2 \%)$ patients. Fives year postoperatively, there were only $9(7.0 \%)$ patients with grade- 4 ossifications in the M6-C artificial disk prosthesis group. In the survival analysis after $\mathrm{HO}$ occurrence, the median survival of the patients was of $28.3 \pm 5.6$ months. The Activ $C$ artificial disk prosthesis group had a statistically longer survival $(49.5 \pm 7.8$ months) than the M6-C disk group.

Conclusion In the present study, $61.4 \%$ of the patients developed $\mathrm{HO}$ at a mean follow-up period of 58.4 months. In the survival analysis after $\mathrm{HO}$ occurrence, the median survival of the patients was of $28.3 \pm 5.6$ months. The Activ $C$ artificial disk prosthesis group had a statistically longer survival ( $49.5 \pm 7.8$ months) than the M6-C disk group.
\end{abstract}

\section{introducción}

La discectomía y fusión cervical anterior (DFCA) es el patrón oro para el tratamiento quirúrgico de la enfermedad degenerativa del disco cervical con un éxito clínico a largo plazo. ${ }^{1,2}$ En los últimos años, la artroplastia de disco cervical $(A D C)$ se ha utilizado ampliamente en pacientes como sustituto para la DFCA. ${ }^{3}$ La introducción de la ADC comenzó con el concepto de preservación del movimiento para tratar la enfermedad degenerativa del disco cervical. La preservación del movimiento imita de modo más fiel el movimiento natural de la columna cervical, y se cree que preserva los segmentos adyacentes de la degeneración a largo plazo en comparación con la DFCA. ${ }^{4}$ Los ensayos controlados aleatorios ${ }^{5-7}$ han informado buenos resultados y una alta satisfacción del paciente después de la ADC.

Sin embargo, se ha reportado ${ }^{8}$ osificación heterotópica $(\mathrm{OH})$ y fusión espontánea después de una ADC, y el mantenimiento del movimiento después de la artroplastia puede verse obstaculizado por el desarrollo de $\mathrm{OH}$. La $\mathrm{OH}$ se define como la formación de hueso fuera del sistema esquelético. Es un fenómeno bien conocido en el campo de la artroplastia total de cadera o de articulación de rodilla, e impide la actividad de los pacientes después de la cirugía. ${ }^{9}$ McAfee et al. ${ }^{10}$ ya describieron y clasificaron el fenómeno de $\mathrm{OH}$ para la artroplastia total de disco lumbar. La tasa de $\mathrm{OH}$ después de ADCs no está clara, pues las tasas informadas varían drásticamente, lo que genera más debate y preocupación en torno a la tasa real y el impacto de la $\mathrm{OH}$. No se han estudiado lo suficiente los efectos a largo plazo de la $\mathrm{OH}$ que resultan en una fusión no intencionada.
El propósito de este estudio fue evaluar la prevalencia de $\mathrm{OH}$ y realizar un análisis de la supervivencia tras la ocurrencia de $\mathrm{OH}$ entre pacientes después de una artroplastia de disco cervical a los 5 años de seguimiento.

\section{Métodos}

\section{Población de pacientes}

El procedimiento de ADC con prótesis Activ C (B. Braun, Sheffield, Reino Unido) y M6-C (Spinal Kinetics, Sunnyvale, CA, EEUU) fue realizado en 127 pacientes, incluidos $65(51,1 \%)$ mujeres y $62(48,8 \%)$ hombres que tenían enfermedad degenerativa del disco cervical sintomática que no respondía al tratamiento conservador entre enero de 2009 y junio de 2011. Los pacientes eran menores de 50 años, con edad media de 38,4 (rango: 18-49) años.

\section{Criterios de elegibilidad y seguimiento}

El criterio de inclusión fue la hernia de disco cervical sintomática en los niveles de C3-C4 a C6-C7 con movilidad preservada $\left(>3^{\circ} \mathrm{y}<11^{\circ}\right)$ en el segmento afectado. Los criterios de exclusión fueron traumatismo, deformidad cifótica, osificación del ligamento longitudinal posterior, o inestabilidad de la columna cervical. Otros criterios de exclusión fueron osteoporosis avanzada, artritis reumatoide, y espondilitis anquilosante (enfermedad de Bekhterev). El tiempo medio de seguimiento fue de 58,4 meses, con un rango de 51 a 66 meses. El protocolo del estudio fue aprobado por el comité de ética local siguiendo la Declaración de Helsinki. ${ }^{11}$ 

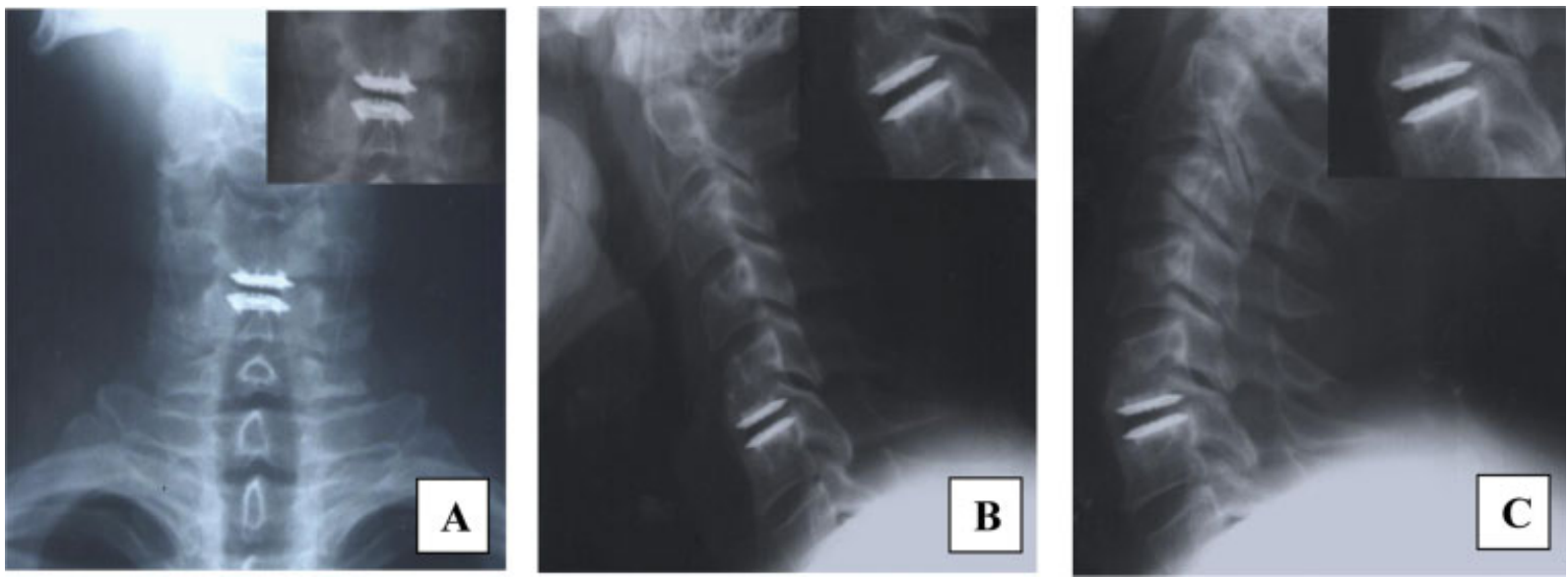

Fig. 1 Paciente del sexo masculino, de 41 años, con enfermedad degenerativa del disco intervertebral C5-C6 tratado mediante procedimiento ADC. Se pueden observar signos claros de $\mathrm{OH}$ de grado III, sin una reducción significativa del movimiento de la prótesis en flexión y extensión a los cinco años de seguimiento: (A) radiografía frontal; (B) radiografía lateral en flexión; (C) radiografía lateral en extensión.

\section{Procedimiento quirúrgico}

Después de un abordaje estándar microquirúrgico anterior de Cloward, se marcó la línea media bajo control fluoroscópico. Se realizaron discectomía y descompresión, y el segmento se separó y se mantuvo así mediante tornillos de retención. Después de probar la altura y el ancho del disco intervertebral por fluoroscopia, se implantó la prótesis adecuada. Se pidió a los pacientes que se levantaran de la cama 24 horas después. Se requirió llevar un collarín no más de una semana.

\section{Evaluación clínica}

Los resultados clínicos de los pacientes incluyeron el índice de discapacidad cervical (IDC), la escala visual analógica (EVA) para el dolor de cuello/brazo, y el puntaje del componente físico (PCF)/puntaje del componente mental (PCM) del cuestionario corto (12) de salud (Short Form [12], SF-12, en inglés).

\section{Evaluación radiológica}

Se utilizaron radiografías cervicales laterales obtenidas en períodos programados antes y después de la cirugía para identificar la $\mathrm{OH}$ (- - Fig. 1). La $\mathrm{OH}$ de algunos casos se confirmó mediante tomografía computarizada (TC) (-Fig. 2). La OH se clasificó según la clasificación de McAfee et al. $^{10}$ Se investigó la tasa de ocurrencia, el período libre de ocurrencia, y la ubicación y el grado de las OHs según los diferentes tipos de prótesis. El comportamiento de la $\mathrm{OH}$ fue observado por dos cirujanos de columna independientes.

\section{Análisis estadístico}

Los análisis de datos se realizaron en los programas Microsoft Office Excel 2016 (Microsoft Corp., Redmond, WA, EEUU) y Statistical Package for the Social Sciences (IBM SPSS Statistics for Windows, IBM Corp., Armonk, NY, EEUU), versión 21.0. Se adoptó un nivel de significancia de 0,05 para todas las pruebas. El análisis estadístico se realizó mediante pruebas $t$ y pruebas de Wilcoxon.

\section{Resultados}

Las características de referencia y las puntuaciones de los pacientes se clasificaron en $\mathrm{OH}$ clínicamente relevante y no
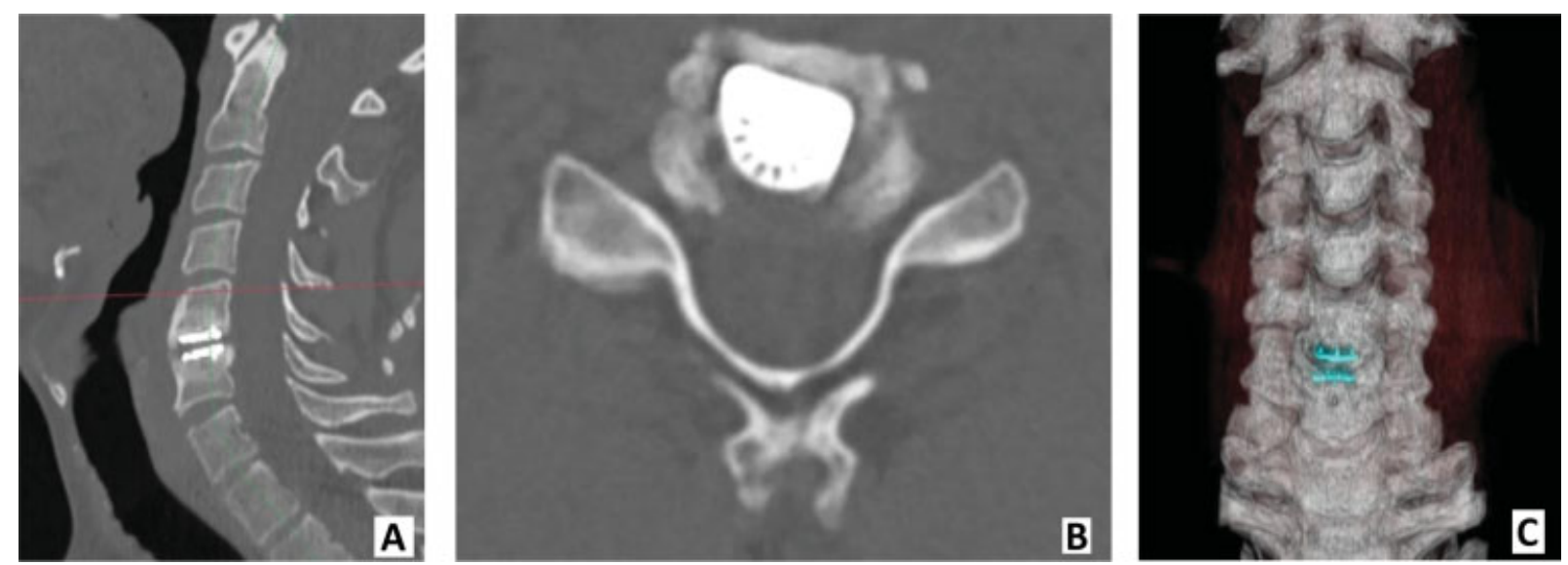

Fig. 2 Tomografías computarizadas (TCS) de OH de grado III (caso clínico anterior): (A) TC sagital; (B) TC axial; (C) TC tridimensional. 
Tabla 1 Características de referencia de los pacientes con $\mathrm{OH}$ de bajo y alto grado 5 años después de la ADC

\begin{tabular}{|l|l|l|l|}
\hline & $\begin{array}{l}\text { Grupo con } \\
\text { OH de bajo } \\
\text { grado }(\boldsymbol{n}=\mathbf{5 6})\end{array}$ & $\begin{array}{l}\text { Grupo con } \\
\text { OH de } \\
\text { alto grado } \\
(\boldsymbol{n}=\mathbf{2 2})\end{array}$ & $p$ \\
\hline Edad media (años) & $39,2 \pm 7,3$ & $38,6 \pm 6,6$ & 0,81 \\
\hline Sexo femenino: $\mathrm{n}(\%)$ & $11(19,6 \%)$ & $16(72,2 \%)$ & 0,07 \\
\hline Altura (cm) & $173,5 \pm 11,3$ & $175,5 \pm 11,2$ & 0,26 \\
\hline Peso (kg) & $75,2 \pm 10,8$ & $79,3 \pm 17,4$ & 0,08 \\
\hline $\begin{array}{l}\text { Índice de masa } \\
\text { corporal* }\end{array}$ & $25,4 \pm 2,5$ & $25,7 \pm 3,8$ & 0,74 \\
\hline $\begin{array}{l}\text { Índice de } \\
\text { discapacidad } \\
\text { cervical }\end{array}$ & 17,9 & 19,9 & 0,17 \\
\hline $\begin{array}{l}\text { Escala visual } \\
\text { analógica para } \\
\text { dolor del cuello }\end{array}$ & 19,2 & 21,1 & 0,37 \\
\hline $\begin{array}{l}\text { Escala visual } \\
\text { analógica para } \\
\text { dolor del brazo }\end{array}$ & 15,4 & 16,9 & 0,44 \\
\hline PCF del SF-12 & 46,8 & 50,3 & 0,96 \\
\hline PCM del SF-12 & 50,5 & $12(54,5 \%)$ & 0,19 \\
\hline $\begin{array}{l}\text { Operación en el nivel } \\
\text { C5-C6: } n \text { (\%) }\end{array}$ & $16(28,5 \%)$ & \\
\hline
\end{tabular}

Abreviaturas: $\mathrm{ADC}$, artroplastia de disco cervical; $\mathrm{OH}$, osificación heterotópica; PCF, puntaje del componente físico; PCM, puntaje del componente mental; SF-12, Short Form (12), cuestionario corto (12) de salud, en inglés.

Nota: *El índice de masa corporal es el peso en kilogramos dividido por el cuadrado de la altura en metros.

relevante presente a los cinco años (-Tabla 1). Estos resultados fueron categorizados para evaluar las tendencias; por lo tanto, las estadísticas no se ejecutaron con estos datos.

En 49 (38,5\%) segmentos tratados, no se detectó OH. Las osificaciones de grado 1 estuvieron presentes en 11 (8,6\%) niveles. Un total de $45(35,4 \%)$ segmentos mostraron $\mathrm{OH}$ de
Tabla 2 Distribución de los diferentes grados de osificación heterotópica según el tipo de prótesis

\begin{tabular}{|l|l|l|l|l|}
\hline & Grado 1 & Grado 2 & Grado 3 & Grado 4 \\
\hline Activ C & $5(3,9 \%)$ & $27(21,3 \%)$ & $8(6,3 \%)$ & $7(5,5 \%)$ \\
\hline M6-C & $6(4,7 \%)$ & $18(14,1 \%)$ & $5(3,9 \%)$ & $2(1,5 \%)$ \\
\hline $\begin{array}{l}\text { Todas las } \\
\text { prótesis }\end{array}$ & $11(8,6 \%)$ & $45(35,4 \%)$ & $13(10,2 \%)$ & $9(7,0 \%)$ \\
\hline
\end{tabular}

grado 2. Las OHs que llevaron a restricciones del rango de movimiento estuvieron presentes en 13 (10,2\%) casos. Cinco años después de la operación, solamente había 9 (7,0\%) pacientes con osificaciones de grado 4 en el grupo con prótesis de disco artificial M6-C.

La osificación localizada anteriormente fue más frecuente que la de localización posterior, pero se debe considerar la dificultad en la detección de la osificación posterior mediante radiografías simples. La distribución de los diferentes grados de $\mathrm{OH}$ según el tipo de prótesis se muestra en la - Tabla 2.

En el análisis de la supervivencia tras la ocurrencia de $\mathrm{OH}$, la mediana de los pacientes fue de 28,3 $\pm 5,6$ meses. El grupo con prótesis de disco artificial Activ C tuvo una supervivencia estadísticamente más larga (49,5 $\pm 7,8$ meses $)$ que el grupo con disco M6-C ( $p=0,003)$ (-Fig. 3).

\section{Discusión}

Es de conocimiento común que la $\mathrm{OH}$ es una complicación posoperatoria inevitable después del procedimiento de ADC, $\mathrm{y}$ puede disminuir el rango de movimiento del segmento operado, lo que va en contra del objetivo fundamental de implantar un disco artificial. ${ }^{12,13}$ Estudios previos reportaron varios resultados sobre la ocurrencia de $\mathrm{OH}$. Lee et al. ${ }^{14}$ relataron que el $78,6 \%$ de los pacientes presentaron $\mathrm{OH}$ en un período de seguimiento medio de 43,4 meses, pero Leung et al. ${ }^{15}$ reportaron un $17,8 \%$ en los pacientes estudiados a los 12 meses de seguimiento. En el

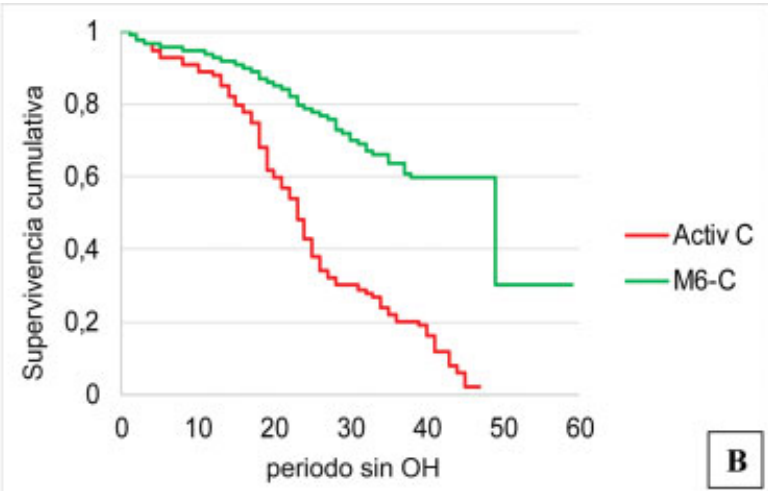

Fig. 3 Análisis de supervivencia tras la ocurrencia de $\mathrm{OH}$ : (A) - la mediana de los pacientes fue de 28,3 \pm 5,6 meses; (B) el grupo con prótesis de disco artificial Activ $C$ tuvo una supervivencia estadísticamente más larga (49,5 $\pm 7,8$ meses) que el grupo con prótesis M6-C ( $p=0,003)$. 
estudio realizado por Yang et al., ${ }^{16}$ la prevalencia fue de hasta un $90 \%$, pero sus resultados se basaron en un seguimiento de 30 años. En la presente investigación, sólo el 38,5\% de los pacientes no mostraron signos de $\mathrm{OH}$ después de la ADC. Existe la hipótesis de que la $\mathrm{OH}$ no es un fenómeno estático, sino un fenómeno dinámico y progresivo, que se ve afectado por el medio ambiente. ${ }^{17}$ De ser así, una duración diferente del seguimiento definitivamente afectaría los resultados finales.

Los factores asociados con la ocurrencia de $\mathrm{OH}$ no se han aclarado. Nunley et al. ${ }^{18}$ mostraron que las razones de probabilidades indicaron que la visita de seguimiento, el sexo masculino, yel puntaje preoperatorio en la EVA para el dolor de cuello están relacionados con el desarrollo de $\mathrm{OH}$, mientras que los cocientes de riesgo indicaron el sexo masculino, la obesidad, la cobertura de la placa terminal, los niveles tratados, y el puntaje preoperatorio en la EVA para el dolor de cuello. Yi et al. ${ }^{19}$ encontraron diferencias en la ocurrencia de $\mathrm{OH}$ según los distintos tipos de prótesis. El uso del Bryan Disc, que es la prótesis menos costreñida, mostró una incidencia significativamente menor de ocurrencia de $\mathrm{OH}$ en comparación con otras prótesis. Los autores ${ }^{19}$ propusieron que las diferencias en el diseño, las propiedades biomecánicas, y el componente específico de articulación de la placa terminal de la prótesis podrían contribuir a la formación de $\mathrm{OH}$. En el presente estudio, la prótesis M6-C también mostró una incidencia significativamente menor de ocurrencia de $\mathrm{OH}$ en comparación con el disco artificial Activ C. Park et al. ${ }^{20}$ descubrieron que la técnica quirúrgica de $\mathrm{ADC}$ influyó en el desarrollo de $\mathrm{OH}$. En este estudio, dos cirujanos de columna realizaron todos los procedimientos de ADC. Sin embargo, tenían diferentes técnicas para recortar las placas terminales. Un cirujano de columna usó una fresa de bola estriada, mientras que el otro usó una fresa tipo diamante. El estudio mostró que el uso de fresa de bola estriada resultó en una tasa significativamente mayor de $\mathrm{OH}$.

Se han discutido varios otros posibles factores causales con respecto a la $\mathrm{OH}$, como no tratar a los pacientes con antiinflamatorios no esteroideos (AINEs) después de diferentes procedimientos quirúrgicos. El uso de AINEs para prevenir la $\mathrm{OH}$ después de un reemplazo total de cadera se reportó anteriormente. ${ }^{21}$ Los protocolos de estudio de ensayos clínicos para ADC realizados por la Administración de Alimentos y Medicamentos de los EEUU (Food and Drug Administration, FDA, en inglés) incluyeron el uso perioperatorio de AINEs en un intento de prevenir la ocurrencia de $\mathrm{OH}$. Un estudio ${ }^{22}$ ha descrito una tendencia hacia la disminución de la formación de $\mathrm{OH}$ en pacientes que usaron AINEs después de ADC en comparación con aquellos que no lo hicieron, pero la diferencia no fue estadísticamente significativa. Los AINEs no se usaron de forma rutinaria en la presente investigación, y estudios adicionales deben evaluar el papel de los AINEs en el desarrollo de OH tras CDA.

Otros factores predisponentes que se han discutido son la edad y el sexo. Anteriormente, se había informado que el sexo masculino se correlacionaba con la formación de $\mathrm{OH}^{23}$ y podría ser un factor contribuyente con respecto a la diferencia observada en la ocurrenciaen comparación con otros informes. Sin embargo, la proporción actual hombre/mujer no fue muy diferente de la de los otros estudios. No hubo relación entre las OHs de alto y bajo grado y la edad o el sexo en el presente estudio.

\section{Limitaciones}

Las limitaciones de este estudio son que sólo se investigaron dos tipos de discos artificiales, y que la población de pacientes era relativamente pequeña. Además, la determinación de $\mathrm{OH}$ fue uniformada sólo por la clasificación de McAfee et al. ${ }^{10}$

\section{Conclusión}

En el presente estudio, el $61,4 \%$ de los pacientes presentaron $\mathrm{OH}$ en un período de seguimiento medio de 58,4 meses. En el análisis de supervivencia tras la ocurrencia de $\mathrm{OH}$, la mediana de los pacientes fue de $28,3 \pm 5,6$. El grupo con prótesis de disco artificial Activ $\mathrm{C}$ tuvo una supervivencia estadísticamente más larga (49,5 $\pm 7,8$ meses) que el grupo con disco M6-C. Esta información es útil para permitir que los cirujanos y los pacientes comprendan mejor la $\mathrm{OH}$ durante el seguimiento. Se necesitan ensayos controlados aleatorios con un seguimiento aún más prolongado para obtener respuestas más definitivas sobre la ocurrencia de $\mathrm{OH}$, los factores asociados con su surgimiento, y su impacto en la movilidad, así como en el resultado clínico.

\section{Aprobación Ética}

La aprobación de la junta de revisión institucional fue obtenida por el comité de ética de la Irkutsk State Medical University (2013-51/5).

\section{Financiamiento}

Los autores no tienen fuentes de financiamiento que declarar.

\section{Conflicto of Intereses}

Los autores no tienen conflicto de intereses que declarar.

\section{Agradecimientos}

Los autores agradecen al personal del Departamento de Cirugía General y Anestesiología de la Irkutsk State Medical University, por su apoyo.

\section{Referencias}

1 Ofluoglu AE, Erdogan U, Aydogan M, Cevik OM, Ofluoglu O. Anterior cervical fusion with interbody cage containing betatricalcium phosphate: Clinical and radiological results. Acta Orthop Traumatol Turc 2017;51(03):197-200. Doi: 10.1016/j. aott.2017.03.001

2 Wang T, Wang H, Liu S, An HD, Liu H, Ding WY. Anterior cervical discectomy and fusion versus anterior cervical corpectomy and fusion in multilevel cervical spondylotic myelopathy: A metaanalysis. Medicine (Baltimore) 2016;95(49):e5437. Doi: 10.1097/ md.0000000000012618 
3 Byvaltsev VA, Stepanov IA, Riew DK. Mid-term to long-term outcomes after total cervical disk arthroplasty compared with Anterior diskectomy and fusion: a systematic review and metaanalysis of randomized controlled trials. Clin Spine Surg 2020;33 (05):192-200. Doi: 10.1097/BSD.0000000000000929

4 Skeppholm M, Svedmark P, Noz ME, Maguire GQJr, Olivecrona H, Olerud C. Evaluation of mobility and stability in the Discover artificial disc: an in vivo motion study using high-accuracy 3D CT data. J Neurosurg Spine 2015;23(03):383-389. Doi: 10.3171/2014.12.spine 14813

5 Gornet MF, Lanman TH, Burkus JK, et al. Cervical disc arthroplasty with the Prestige LP disc versus anterior cervical discectomy and fusion, at 2 levels: results of a prospective, multicenter randomized controlled clinical trial at 24 months. J Neurosurg Spine 2017;26(06):653-667. Doi: 10.3171/2016.10.spine16264

6 Rožanković M, Marasanov SM, Vukić M Cervical Disk Replacement With Discover Versus Fusion in a Single-Level Cervical Disk Disease: A Prospective Single-Center Randomized Trial With a Minimum 2-Year Follow-up. Clin Spine Surg 2017;30(05): E515-E522. Doi: 10.1097/bsd.0000000000000170

7 Sundseth J, Fredriksli OA, Kolstad F, et al; NORCAT study group. The Norwegian Cervical Arthroplasty Trial (NORCAT): 2-year clinical outcome after single-level cervical arthroplasty versus fusion-a prospective, single-blinded, randomized, controlled multicenter study. Eur Spine J 2017;26(04):1225-1235. Doi: 10.1007/s00586-016-4922-5

8 Zeng J, Liu H, Chen H, et al. Comparison of Heterotopic Ossification After Fixed- and Mobile-Core Cervical Disc Arthroplasty. World Neurosurg 2018;120:e1319-e1324. Doi: 10.1016/j. wneu.2018.09.075

9 Arnold PM, Anderson KK, Selim A, Dryer RF, Kenneth Burkus J. Heterotopic ossification following single-level anterior cervical discectomy and fusion: results from the prospective, multicenter, historically controlled trial comparing allograft to an optimized dose of rhBMP-2. J Neurosurg Spine 2016;25(03):292-302. Doi: 10.3171/2016.1.spine15798

10 McAfee PC, Cunningham BW, Devine J, Williams E, Yu-Yahiro J. Classification of heterotopic ossification (HO) in artificial disk replacement. J Spinal Disord Tech 2003;16(04):384-389

11 Williams JR. The Declaration of Helsinki and public health. Bull World Health Organ 2008;86(08):650-652

$12 \mathrm{Tu} \mathrm{TH}, \mathrm{Wu}$ JC, Huang WC, et al. Heterotopic ossification after cervical total disc replacement: determination by CT and effects on clinical outcomes. J Neurosurg Spine 2011;14(04):457-465. Doi: $10.3171 / 2010.11$.spine10444
13 Mehren C, Suchomel P, Grochulla F, et al. Heterotopic ossification in total cervical artificial disc replacement. Spine 2006;31(24): 2802-2806. Doi: 10.1097/01.brs.0000245852.70594.d5

14 Lee SE, Jahng TA, Kim HJ. Correlation between cervical lordosis and adjacent segment pathology after anterior cervical spinal surgery. Eur Spine J 2015;24(12):2899-2909. Doi: 10.1007/ s00586-015-4132-6

15 Leung C, Casey AT, Goffin J, et al. Clinical significance of heterotopic ossification in cervical disc replacement: a prospective multicenter clinical trial. Neurosurgery 2005;57 (04):759-763, discussion 759-763. Doi: 10.1093/neurosurgery/ 57.4.759

16 Yang H, Lu X, Yuan W, Wang X, Chen D, Zhao D. Artificial disk replacement in the treatment of degenerative cervical disk disorder: a 30-year follow-up study. Spine 2014;39(19): 1564-1571. Doi: 10.1097/brs.0000000000000484

17 Jin YJ, Park SB, Kim MJ, Kim K-J, Kim H-J. An analysis of heterotopic ossification in cervical disc arthroplasty: a novel morphologic classification of an ossified mass. Spine J 2013;13(04):408-420. Doi: 10.1016/j.spinee.2012.11.048

18 Nunley PD, Cavanaugh DA, Kerr EJ III, et al. Heterotopic Ossification After Cervical Total Disc Replacement at 7 YearsPrevalence, Progression, Clinical Implications, and Risk Factors. Int J Spine Surg 2018;12(03):352-361. Doi: 10.14444/5041

19 Yi S, Kim KN, Yang MS, et al. Difference in occurrence of heterotopic ossification according to prosthesis type in the cervical artificial disc replacement. Spine 2010;35(16): 1556-1561. Doi: 10.1097/brs.0b013e3181c6526b

20 Park JH, Rhim SC, Roh SW. Mid-term follow-up of clinical and radiologic outcomes in cervical total disk replacement (Mobi-C): incidence of heterotopic ossification and risk factors. J Spinal Disord Tech 2013;26(03):141-145. Doi: 10.1097/ BSD.0b013e31823ba071

21 Sodemann B, Persson PE, Nilsson OS. Prevention of heterotopic ossification by nonsteroid antiinflammatory drugs after total hip arthroplasty. Clin Orthop Relat Res 1988;(237):158-163

$22 \mathrm{Tu} \mathrm{TH}$, Wu JC, Huang WC, et al. Postoperative nonsteroidal antiinflammatory drugs and the prevention of heterotopic ossification after cervical arthroplasty: analysis using CT and a minimum 2-year follow-up. J Neurosurg Spine 2015;22(05): 447-453. Doi: 10.3171/2014.10.spine14333

23 Yi S, Shin DA, Kim KN, et al. The predisposing factors for the heterotopic ossification after cervical artificial disc replacement. Spine J 2013;13(09):1048-1054. Doi: 10.1016/j. spinee.2013.02.036 